\title{
Lenalidomide treatment of myelodysplastic syndromes with chromosome 5q deletion: Results from the National Registry of the Italian Drug Agency
}

\author{
Francesco Arcioni ${ }^{1}$ | Andrea Roncadori ${ }^{2}$ | Valeria Di Battista ${ }^{1}$ (D) | Sante Tura ${ }^{3}$ | \\ Anna Covezzoli $^{2}$ | Sante Cundari ${ }^{4}$ | Cristina Mecucci ${ }^{1}$ | on behalf of MORE Study Centres*
}

${ }^{1}$ Department of Medicine, Institute of Hematology and Center for HematoOncology Research (C.R.E.O.), University of Perugia, Perugia, Italy

${ }^{2}$ CINECA Interuniversity Consortium, Bologna, Italy

${ }^{3}$ University of Bologna, Bologna, Italy

${ }^{4}$ Celgene International Sarl, Milano, Italy

Correspondence

Cristina Mecucci, Institute of Hematology and Center for Hemato-Oncology Research (C.R.E.O.), Perugia, Italy.

Email: cristina.mecucci@unipg.it

Funding information

This study was entirely supported by Celgene Italia.

\begin{abstract}
Objective: The most typical cytogenetic aberration in myelodysplastic syndromes is del $(5 q)$, which, when isolated, is associated with refractory anaemia and good prognosis. Based on high rates of erythroid response and transfusion independence, Lenalidomide (LEN) became the standard treatment. This multi-centre study was designed to supplement Italian Registry data on LEN by addressing prescription, administration appropriateness, haematological and cytogenetic responses and disease evolution.
\end{abstract}

Methods: MORE study was an observational, non-interventional, multi-centre, retrospective and prospective study. Cases were recruited from 45 Haematological Centres throughout Italy. Data were collected from the Italian National Registry for Lenalidomide administration and supplemented by a MORE data form.

Results: Data from 190/213 patients were analysed. In all, 149 had been diagnosed by conventional cytogenetics (GROUP A) and 41 only by FISH (GROUP B). Overall erythroid response was obtained in $92.8 \%$ of cases. Overall cytogenetic remission was achieved in $22.6 \%$ of cases. Disease progression occurred in $15.6 \%$ of cases. Clonal cytogenetic evolution characterised progression to $\mathrm{AML}$ but not to higher risk MDS.

Conclusions: Erythroid response to Lenalidomide was similar in MDS with isolated del $(5 q)$ and with del $(5 q)$ plus one anomaly. Progression to AML or higher risk MDS showed different cytogenetic features.

KEYWORDS

del(5q), lenalidomide, myelodysplastic syndromes, registry study

\section{1 | INTRODUCTION}

The most typical cytogenetic aberration in de novo myelodysplastic syndromes (MDS) is isolated del(5q), an interstitial deletion within

Arcioni, Roncadori and Di Battista equally contributed to this study.

*See Appendix for MORE Study Centres.

ClinicalTrials.gov: NCT01347944. the chromosome 5 long arm, which is the hallmark of refractory anaemia, macrocytosis, normal or high platelet count, erythroid hypoplasia, non-lobulated megakaryocytes, and relatively good prognosis. LEN was recognised as efficacious therapy as it suppresses del( $5 q)$ clonal cells and acts on the erythroid compartment to improve haemoglobin levels, leading to an $83 \%$ erythroid response rate and durable transfusion independence. ${ }^{1}$ Cytogenetic response rates were much higher in patients with isolated $\operatorname{del}(5 q)$ than in patients 
TAB LE 1 Demographics, MDS classification according to IPSS, WPSS, FAB and WHO 2008 and cytogenetic characteristics of patients

\begin{tabular}{|c|c|c|}
\hline & $\begin{array}{l}\text { Group A } \\
149 \text { patients }\end{array}$ & $\begin{array}{l}\text { Group B } \\
41 \text { patients }\end{array}$ \\
\hline \multicolumn{3}{|l|}{ Gender } \\
\hline Female & $105(70,5 \%)$ & $25(61,0 \%)$ \\
\hline Male & $44(29,5 \%)$ & $16(39,0 \%)$ \\
\hline \multicolumn{3}{|l|}{ Age } \\
\hline Median & 75 & 71 \\
\hline Range & [38; 95] & {$[41 ; 87]$} \\
\hline \multicolumn{3}{|l|}{ FAB Classification } \\
\hline Refractory Anaemia & $105(70.5 \%)$ & $24(58,5 \%)$ \\
\hline $\begin{array}{l}\text { Refractory Anaemia with } \\
\text { ringed sideroblasts }\end{array}$ & $2(1.3 \%)$ & 0 \\
\hline $\begin{array}{l}\text { Refractory Anaemia with } \\
\text { excess of blasts }\end{array}$ & $17(11.4 \%)$ & $3(7.3 \%)$ \\
\hline Missing & $25(16,8 \%)$ & $14(34.1 \%)$ \\
\hline \multicolumn{3}{|l|}{ WHO Classification } \\
\hline Refractory Anaemia & $9(6 \%)$ & $3(7.3 \%)$ \\
\hline $\begin{array}{l}\text { Refractory Anaemia with } \\
\text { excess of blasts-1 }\end{array}$ & $15(10.1 \%)$ & $3(7.3 \%)$ \\
\hline $\begin{array}{l}\text { Refractory Anaemia with } \\
\text { excess of blasts- } 2\end{array}$ & $1(0.7 \%)$ & 0 \\
\hline $\begin{array}{l}\text { Refractory cytopenia } \\
\text { with multilineage } \\
\text { dysplasia }\end{array}$ & $25(16.8 \%)$ & $5(12.2 \%)$ \\
\hline MDS-5q & 79 (53\%) & $26(63.4 \%)$ \\
\hline MDS-Unclassificable & $3(2 \%)$ & 0 \\
\hline Missing & $17(11.4 \%)$ & $4(9.8 \%)$ \\
\hline \multicolumn{3}{|l|}{ IPSS Classification } \\
\hline Low & $69(46.3 \%)$ & $20(48.8 \%)$ \\
\hline Intermediate -1 & $80(53.7 \%)$ & $21(51.2 \%)$ \\
\hline \multicolumn{3}{|l|}{ WPSS Classification } \\
\hline Very low & $7(4.7 \%)$ & $2(4.9 \%)$ \\
\hline Low & $51(34.2 \%)$ & $12(29.3 \%)$ \\
\hline Intermediate & $16(10.7 \%)$ & 2 (4.9\%) \\
\hline High & $8(5.4 \%)$ & $1(2.4 \%)$ \\
\hline Missing & $67(45 \%)$ & $24(58.5 \%)$ \\
\hline \multicolumn{3}{|l|}{ Karyotype (only for Group A) } \\
\hline $5 q$ isolated & 122 (81.9\%) & NA \\
\hline $5 q$ not isolated & $25(16.8 \%)$ & NA \\
\hline Complex & $2(1.3 \%)$ & NA \\
\hline \multicolumn{3}{|l|}{ LEN cycles (median) } \\
\hline & $12[5 ; 24]$ & $13[4 ; 34]$ \\
\hline
\end{tabular}

LEN, Lenalidomide.

with del(5q) in complex karyotypes. ${ }^{2}$ The European MDS 004 study confirmed these data, recommending $10 \mathrm{mg}$ LEN every 21 days in 28-day cycles. ${ }^{3}$
After LEN was approved by the Food and Drug Administration (USA) and by the European Medicines Agency, administration under the Italian National Health Service was limited to patients with $\operatorname{del}(5 q)$, whether isolated or not, and low or intermediate-1 risk MDS (LOW or INT-1 according to the IPSS score). ${ }^{4}$ They were enrolled in the Italian National Drug Agency Registry (AIFA, Agenzia Italiana Farmaco), which monitors administration of drugs that are still under investigation.

The present multi-centre study was designed to supplement Registry data on LEN by addressing prescription and administration appropriateness, haematological and cytogenetic responses and disease evolution.

\section{METHODS}

\section{1 | Setting}

Inclusion Criteria for the AIFA LEN Registry were as follows: LOW or INT-1 MDS; transfusion-dependent anaemia (at least 2 units of packed RBCs in 8 weeks prior to starting LEN treatment); $5 \mathrm{q}$ deletion, whether isolated or associated with other chromosomal abnormalities.

\section{2 | Study design}

This observational, non-interventional, multi-centre, retrospective/prospective cohort study was registered as MORE (ClinicalTrials.gov NCT01347944) and designed as follows: collection of retrospective data on patients that had been enrolled in the Registry from October 31, 2008 to May 20, 2010; collection of prospective data as patients were recruited to the study from May 21, 2010 to June 13, 2012 (Figure S1); integration of both databases using a new MORE data form. All subjects in the Registry, who had undergone at least one cycle of LEN, were included in the MORE study.

The present study included 45 Italian Haematological Centres: 22 from northern Italy, 11 middle and 12 southern (Figure S2). The Ethics Committee of each participating centre approved the study.

All data were independently collected from AIFA Registry through CINECA, a non-profit Inter-university Consortium for data collection, processing and statistical analysis. Data were monitored by CRO (MeDePha, Via Aosta 4/A, 20155, Milano), a data management centre that organised, and was responsible for, inter-centre contacts, checked patient electronic Continuous Reinforcement Schedules (e-CRFs) and replied to e-Queries. A Scientific Steering Committee focused on centre compliance with procedures and checked data analysis.

Patients were divided into Group A, who underwent conventional cytogenetic testing, and Group B, who underwent only FISH (Table 1). Two groups were analysed separately according to LEN dosage: $10 \mathrm{mg} / \mathrm{d}$ vs $5 \mathrm{mg} / \mathrm{d}$ (Table S1).

The primary study objectives were to determine prescription and administration appropriateness and to assess clinical, 
haematological and cytogenetic responses to LEN in-depth, according to International Working Group criteria. ${ }^{5}$

Secondary objectives were to evaluate diagnostic approaches; to monitor cytogenetic and haematological changes during the course of disease; to identify subgroups with significant prognostic features and monitor LEN safety and tolerability.

Data analysis was conducted at predetermined time-points: after 4-6 cycles of treatment; after 8-12 cycles; at last follow-up and/or at the end of treatment. Only the cases with complete information at the three time-points entered both univariate and multivariate analyses. A total of 9 clinical variables were considered: bone marrow blasts, MCV, haemoglobin level, neutrophil count, ferritin levels, platelet count, Abnormal Localisation of Immature Precursors (ALIP), megakaryocytic dysplasia and bone marrow fibrosis (Table S2). For safety and toxicity assessment, frequency of patients with any adverse events such as neutropenia, thrombocytopenia or infections, early withdrawal, hospitalisations were reported.

\section{3 | Statistical Analysis}

Descriptive statistics (mean, SD, median, range and inter-quartile range) were calculated for all continuous variables. Frequency distributions were calculated for categorical variables (disease, risk category, haematological and cytogenetic profiles over time, response to LEN, disease progression). The $t$ test for continuous variables and the chi-square test for categorical variables were used to analyse inter-group differences (unless otherwise stated, all $P$ values are intended as two-tailed). Kaplan-Meier survival curves were calculated using time-to-event variables and the log-rank test for group comparison. Univariate logistic regression analyses evaluated some baseline variables and prognostic factors including blast and platelet counts, cytogenetic complexity at diagnosis, erythroid and/or cytogenetic response, to identify the prognostic factors associated with evolution to AML or higher risk MDS. Significant variables in univariate analysis (at alpha 0.1) were analysed in multivariate logistic regression analysis.

The LOCF (Last Observation Carried Forward) method was applied to handle missing data.

\section{4 | IT infrastructure and software}

The web-based system for data collection was the CINECA $A X M R \circledast$ (Advanced Extended Multicentre Research) technology that was designed to manage clinical research processes. Data management (DB freezing, intermediate tables, views and materialised views in support of the analysis) was carried out using PL/ SQL Developer (Oracle Corporation database), which is based on the PL/SQL (Procedural Language/Structured Query Language) program.

Data analysis was performed using PL/SQL Developer and $\mathrm{R}$ open-source software, which is specific for statistical calculations and charting.

\section{3 | RESULTS}

\subsection{Study size, grouping and bias}

In all, 213 patients were included in the registry during the study period (56 up to May 20, 2010 who provided retrospective data and 134 afterwards who yielded both prospective and retrospective data).

Totally, 190/213 patients (M:F 60:130) were eligible for the study as they satisfied all inclusion criteria. At inclusion in the registry, 56 patients had been pre-treated with LEN, and the previous cycles were added by MORE integration. Starting doses of LEN were extremely heterogeneous; however, we were able to distinguish two groups: $59 \%$ of patients received $10 \mathrm{mg}$ daily (in 21day cycles or continuously) and $27 \%$ of patients were given $5 \mathrm{mg}$ (in 21-day cycles or continuously) (Table S1). Moreover, 128/190 cases (62\%) stopped LEN treatment during the observation period (Table S3). Patients who had undergone only FISH testing (Group B) (41 patients, median age 71, 10 pre-treated) were analysed separately from those who underwent a full cytogenetic evaluation (Group A) (149 patients, median age 75, 46 pre-treated). On a total of $18.1 \%$ of patients with non-isolated $5 q-, 1.3 \%$ had a complex karyotype, $16.8 \%$ one additional chromosomal abnormality. No significant inter-group ( $A$ vs $B$ ) differences emerged in patient demographics, disease and risk categories or median time from diagnosis to LEN treatment (Table 1).

\section{2 | Erythroid response}

The complete erythroid response rate was $74.6 \%$ in group $A$ and $78.6 \%$ in group $B$ after $4-6$ cycles, rising to $85.8 \%$ and $88.9 \%$, respectively, after $8-12$ cycles. The partial response rate was $11.5 \%$ in group $A$ and $10.7 \%$ in group B after $4-6$ cycles, falling to $6.2 \%$ and $7.4 \%$, respectively, after $8-12$ cycles as patients achieved complete response (Figures 1 , Table S4). In univariate analysis number of cycles $>6$, platelet count $>100000$ and ALIP were significant both for complete and partial response; $\mathrm{Hb}$ and blast count resulted significant only for overall response $(P<.05)$ (Tables $S 5$ and S6.). Only the duration of therapy reached the statistical significance in multivariate analysis $(P<.001)$ (Tables S7 and S8). No differences in overall and complete responses emerged in two treatment schedules.

\section{3 | Cytogenetic response}

Cytogenetic response was analysed and monitored only in patients who had undergone conventional cytogenetics for $5 q$ - diagnosis (Group A). After 4-6 cycles, the complete response rate was $7.8 \%$, whereas the partial response rate was $2.4 \%$. After $8-12$ cycles, the complete response rate rose to $13 \%$ and the partial to $9.6 \%$ (Figure 2, Table S4). Starting dosage at $10 \mathrm{mg}$ LEN daily $(P<.001)$ and erythroid dysplasia $(P<.05)$ significantly correlated with overall cytogenetic response. Only the starting dosage retains the statistical 
(A)

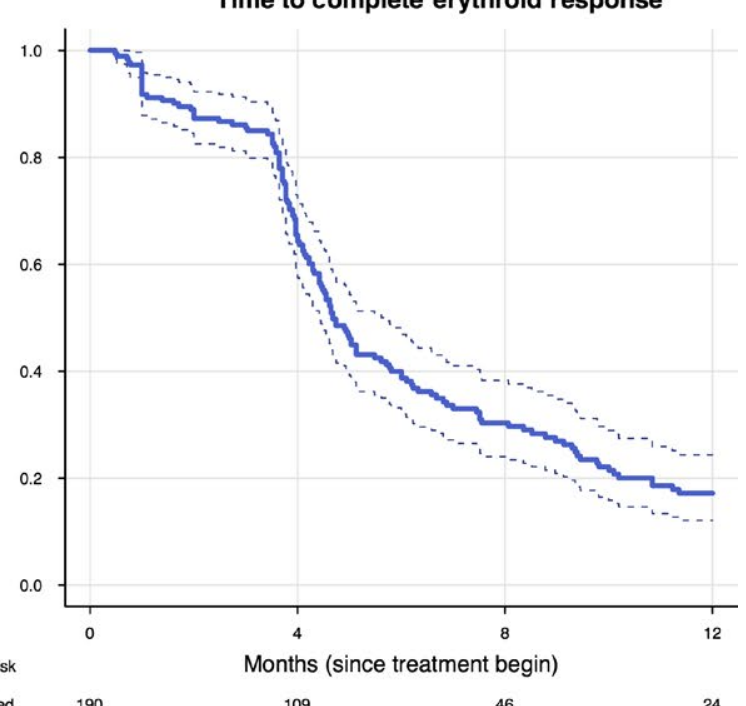

Treated 190

109

Time to any erythroid response

(B)

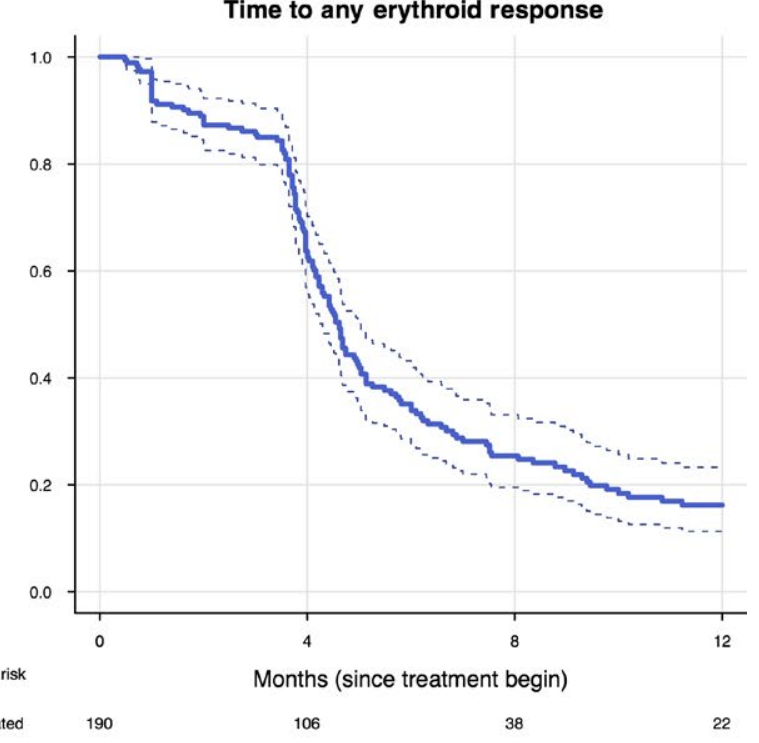

Time to complete erythroid response curves by karyotype at baseline (GROUP A)

(C)

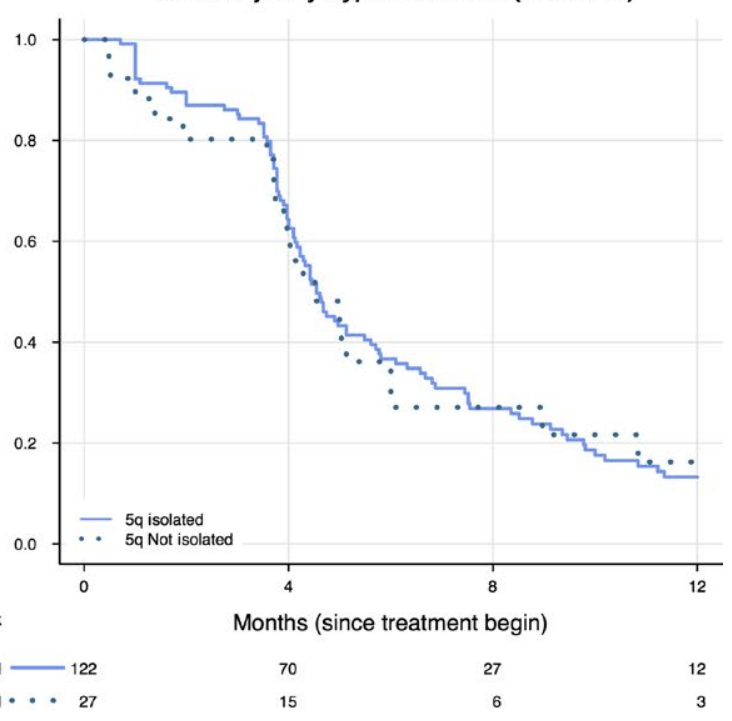

FIGURE 1 Time to development of complete (A) and overall erythroid response $(B)$ in all patients (groups $A$ and $B$ ). Time to complete erythroid response, only Group A ( $5 q$ isolated vs $5 q$ not isolated) (C) since treatment begin [Colour figure can be viewed at wileyonlinelibrary.com]

significance in multivariate analysis (Table 3). None of these factors resulted significant for complete cytogenetic response in univariate analysis (data not shown). Within the $10 \mathrm{mg}$ Group 60\% of cases showed cytogenetic response at 24 months therapy (Figure 2C). However, dosage did not impact both leukaemia free survival (LFS) and overall survival (OS) (Figure S6).

\section{4 | Disease progression}

During the 44-month observation period (range: 0.5-237), disease progression occurred in $15.6 \%$ of cases: $18 / 190$ cases (9.5\%) developed AML (Table 2 ) and 12 cases (6.1\%) progressed to higher risk MDS (Table 3). We analysed separately cases developing AML and those progressing to higher risk MDS. Both subgroups were negative for any cytogenetic response to LEN treatment (Table 2). In 7/13 Group A patients, AML diagnosis corresponded to clonal cytogenetic progression and acquisition of a complex karyotype, whereas complex cytogenetics never appeared in the subgroup evolving to higher risk MDS; in the last group, the del( $5 q$ ) karyotype of diagnosis remained unchanged. In univariate analysis, cytogenetic and erythroid response did not influence progression to $A M L$ or higher risk MDS (Figures S3 and S4). Significant differences emerged in time to progression (AML median 25 months, range 7-89; MDS median 54 months, range 15-102; $P$.028) and number of LEN cycles (median: 7.5 for AML and 19.5 for higher risk MDS, $P$.010). Other clinical and biological variables assessed in the present study include blast and platelet counts, cytogenetic complexity at diagnosis, erythroid and/ or cytogenetic response and time from MDS diagnosis and inclusion in the Registry. The only factor which achieved significance in our study was a blast count of $>5 \%(P .010)$ in both univariate analysis (Figure S5) and multivariate analysis $(P<.05)$ (Table S9).

\subsection{LEN safety and toxicity}

During treatment, most patients had slight to moderate neutropenia (75\%; grade 3\%-4 59\%) and thrombocytopenia (62\%; grade 3\%-4 21\%). Grade 3-4 Neutropenia led to drug discontinuation in fewer than $50 \%$ of cases and was treated with G-CSF in fewer than $10 \%$. The incidences of neutropenia and thrombocytopenia were greater in the first 6 months of treatment. Infections (21\%) were mostly upper respiratory tract infections. During the observation period, 13 patients $(31.7 \%)$ were hospitalised because of infection. Five patients with platelet counts of $>100000 / \mathrm{mmc}$ and haemoglobin $>10 \mathrm{~g} / \mathrm{dL}$ in the early phase of treatment had deep venous thrombosis leading to LEN withdrawal. In one patient with HCV-related cirrhosis, liver toxicity led to early withdrawal after only 1 LEN cycle. 
(A)

Time to complete cytogenetic response

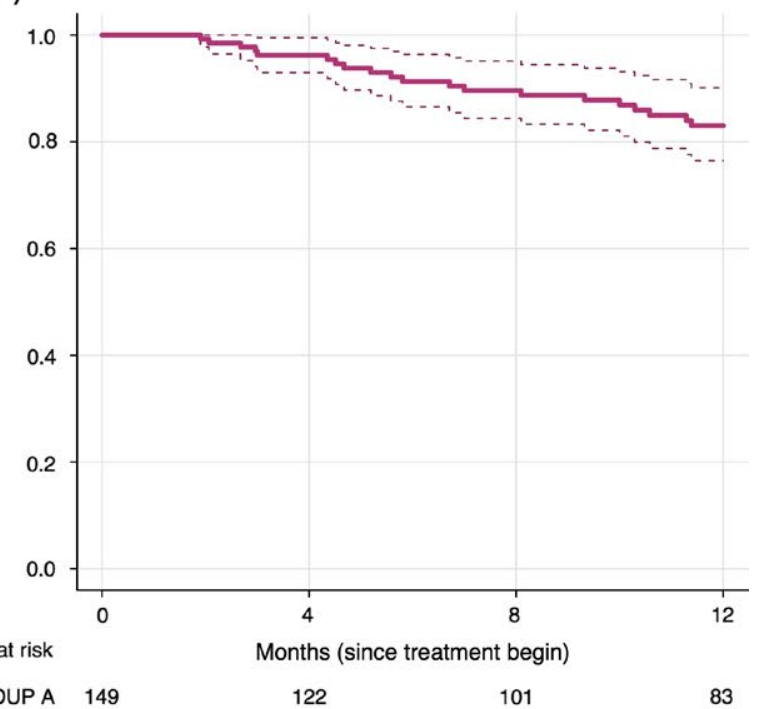

(B)
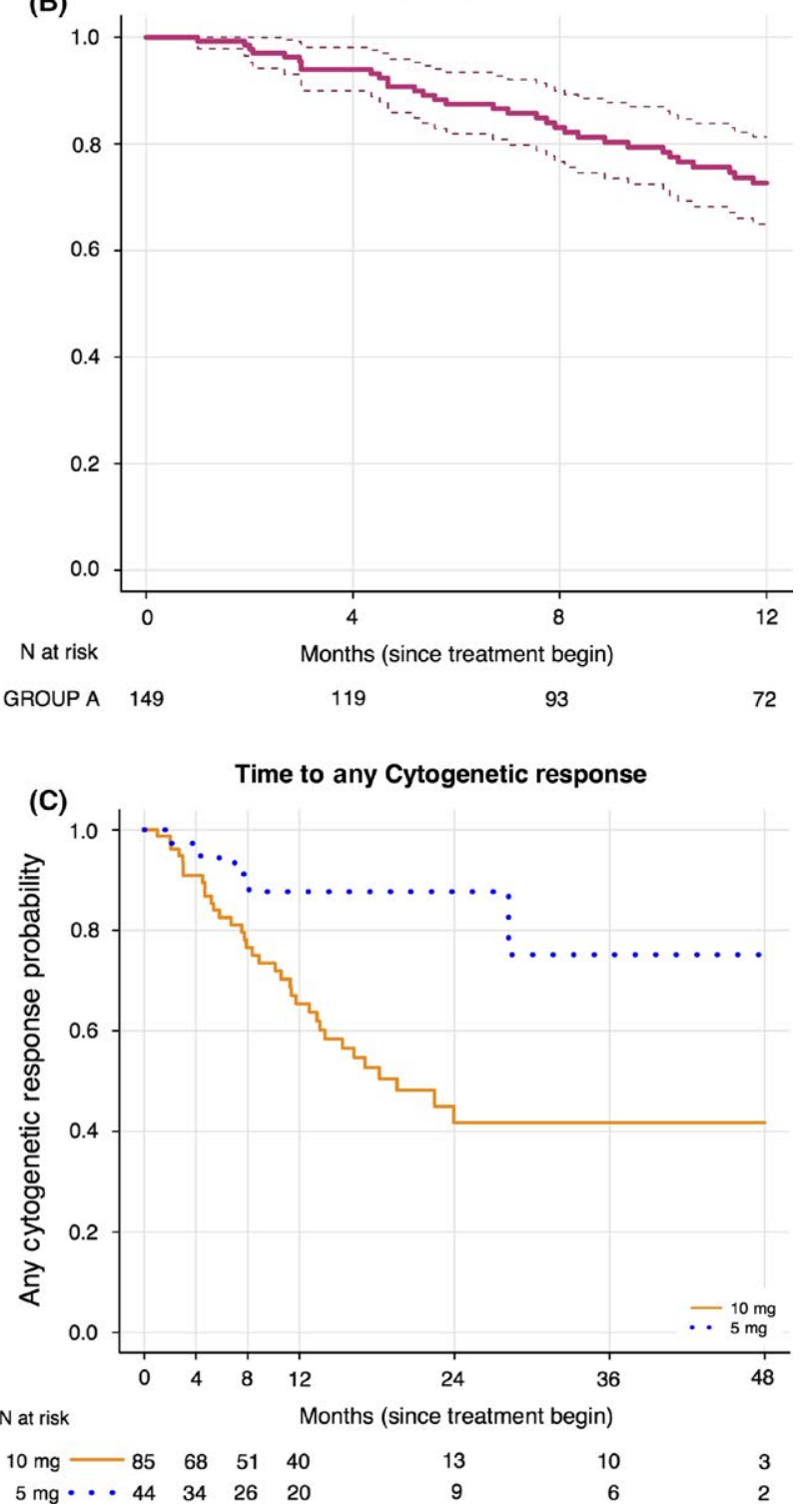

FIGURE 2 Time to development of complete (A) and overall cytogenetic response (B) in Group A (data are referred to first 24 mo of treatment); cytogenetic response by treatment group for the first 48 mo of treatment (C) [Colour figure can be viewed at wileyonlinelibrary.com]

\section{4 | DISCUSSION}

As far as we know, this is the first study on LEN use in the framework of a national registry, in which Haematology Units throughout Italy participated. Italian haematologists correctly selected patients and managed LEN administration. MORE integration data indicated a high response rate, supporting appropriate diagnosis and monitoring.

High erythroid response rates confirmed LEN had exerted its well-known effect in del(5q) MDS. ${ }^{1-3,6-8}$ This effect has been emphasised in a heterogeneous cohort of 716 MDS patients with $71 \%$ overall erythroid response after 3 cycles of LEN; $83 \%$ of responders had MDS with del(5q). ${ }^{9}$ The drop of haemoglobin level we observed at the last follow-up was likely due to the relative high number of LEN suspension (98 cases) or disease progression (30 cases). Haematological response was independent of cytogenetic remission, as demonstrated by the lower cytogenetic response rate compared to the erythroid, similarly to previous published series. ${ }^{3,10}$

Overall cytogenetic response in this study was very low compared to the previous reported data. Indeed, major limitations of this study were treatment schedules that were heterogeneous for duration, continuous vs intermittent administration and individual dosage adjustments. No significant differences were found considering overall response after 6 cycles $(P=.8)$. Instead LEN dosage, similarly to that reported in pivotal European MDS-004, ${ }^{3}$ influenced cytogenetic response as the $10 \mathrm{mg}$ initial dosage predicted cytogenetic response. Notably in the group of patients treated by long-standing $10 \mathrm{mg}$ dosage, cytogenetic response increased to $60 \%$.

The impact of $5 q$ - plus another anomaly on the erythroid response rate in low and intermediate-1 risk MDS has been debated. A retrospective cytogenetic multi-centre Spanish study found the erythroid response differed significantly if one aberration in addition to the $5 q$ - was present, but the IPSS risk category was not evaluated. ${ }^{11}$ In the present study, a good response rate was observed even when an additional chromosomal change accompanied del( $5 q)$, thus emphasising similarities between isolated $\operatorname{del}(5 q)$ and $\operatorname{del}(5 q)$ plus one additional anomaly, as recently recognised by the WHO $2016 .{ }^{12}$ Remarkably, in our series, all cases belonged to LOW or INT1 IPSS category. This may be relevant considering that around $43 \%$ of LOW and INT1 MDS without del(5q) responded to LEN. ${ }^{13}$ Consequently, LEN appears optimal treatment for LOW and INT1 MDS with isolated del $(5 q)$, or del $(5 q)$ plus one more change.

Overall, evolution rate $(15,6 \%)$ in this Registry study was lower than the rate of $25,4 \%$ in the MDS-004 European study ${ }^{3}$ although they had comparable observation time ( 44 months vs 35.5 months in the LEN $5 \mathrm{mg}$ group and 36.9 months in the LEN $10 \mathrm{mg}$ group) and baseline cytogenetics. In his update of 148 cases, including 16,9\% 


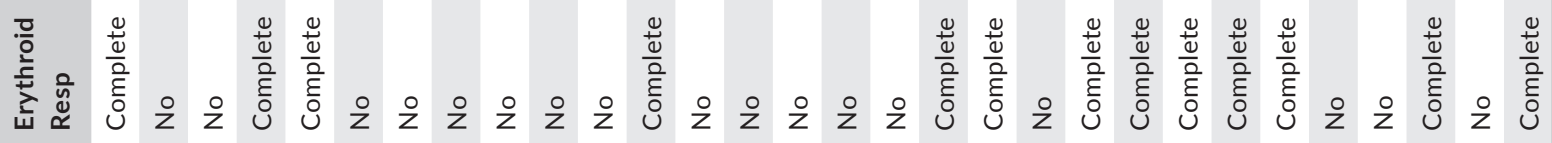

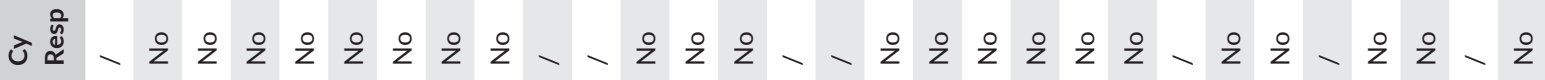

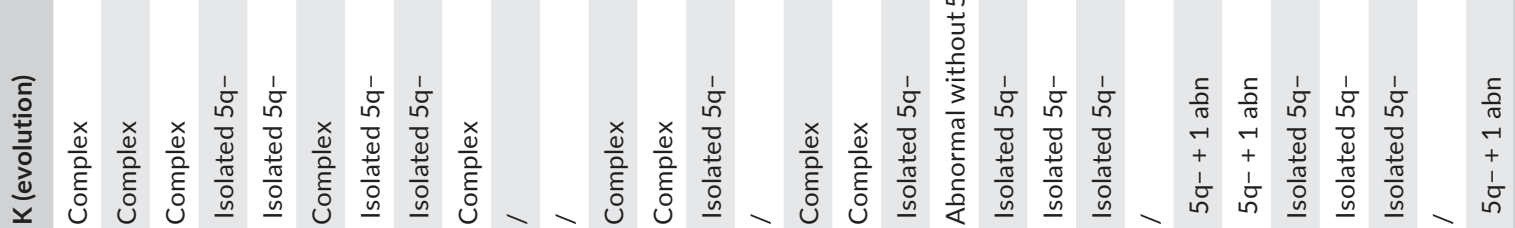

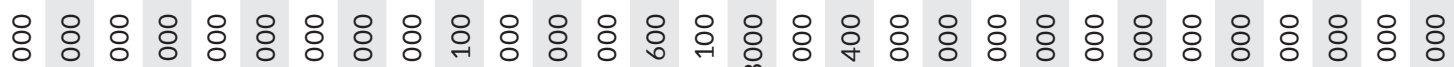
দa

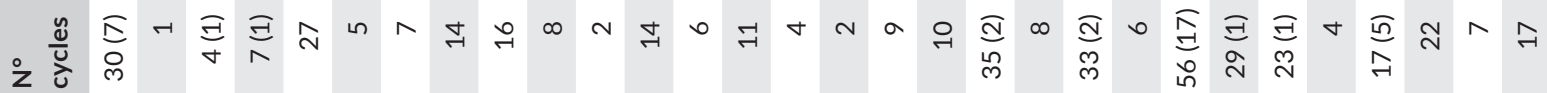

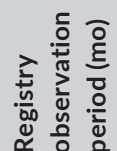

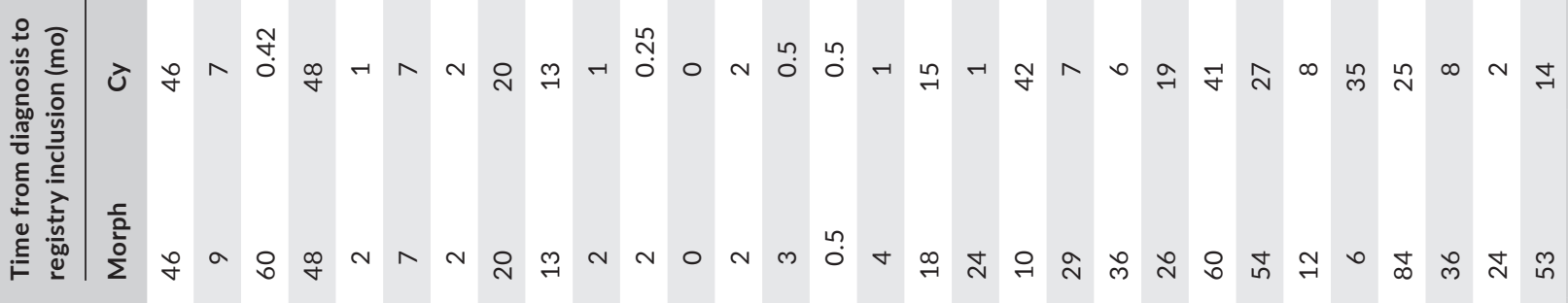

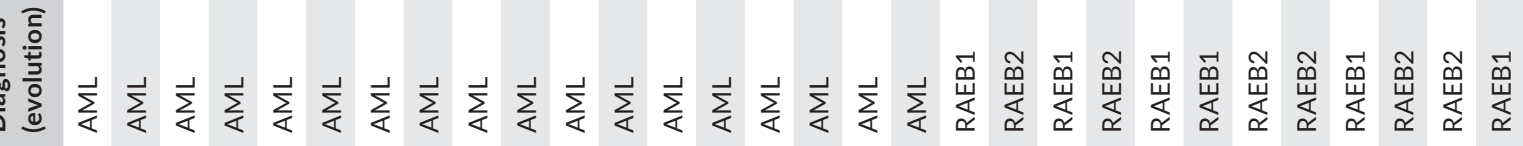

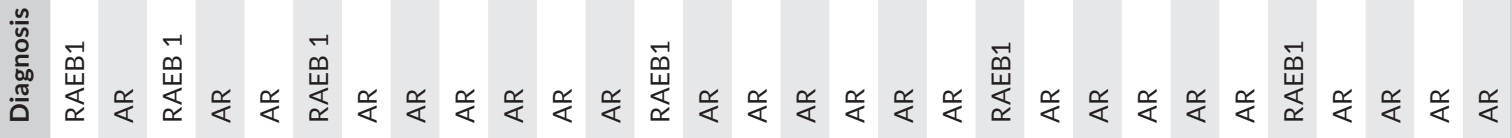

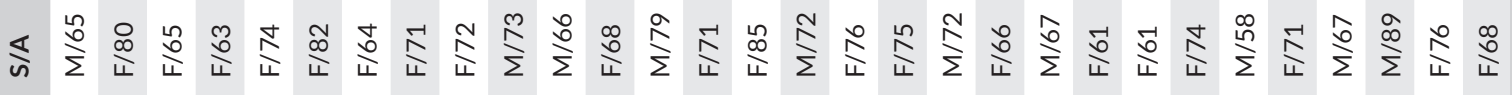

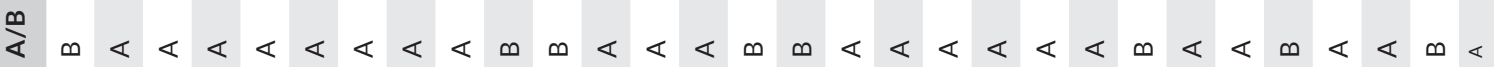

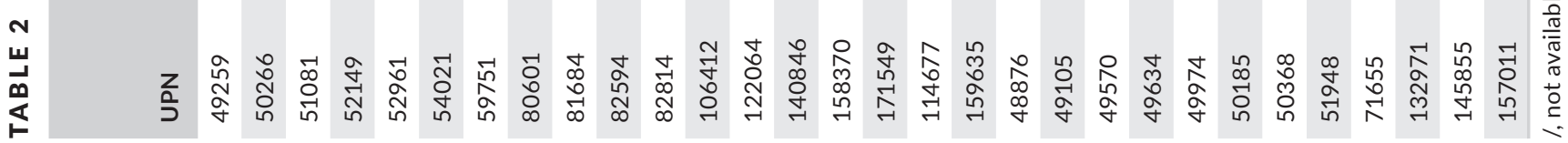


TAB LE 3 Factors associated with cytogenetic response (any response, both complete and partial) in group $A$

\begin{tabular}{|llr|}
\hline & OR $(95 \% \mathrm{Cl})$ & P value \\
\hline Univariate analysis & & \\
\hline Blasts & $1.10[0.93-1.29]$ & .261 \\
\hline Granulocytic dysplasia & $0.56[0.25-1.27]$ & .164 \\
\hline Erythroid dysplasia & $5.35[1.08-26.4]$ & .039 \\
\hline Megakaryocytic dysplasia & $2.77[0.93-8.24]$ & .066 \\
\hline Platelets $(\geq 100$ 000) & $0.58[0.20-1.67]$ & .312 \\
\hline ALIP & $0.67[0.06-7.55]$ & .749 \\
\hline MCV & $0.98[0.94-1.01]$ & .184 \\
\hline Bone marrow fibrosis & $0.41[0.07-2.23]$ & .302 \\
\hline Hb (g/dL) & $0.98[0.80-1.20]$ & .871 \\
\hline Karyotype $(5 q-$ isolated) & $2.04[0.65-6.35]$ & .219 \\
\hline IPSS (Low risk) & $0.81[0.37-1.76]$ & .597 \\
\hline Initial dosage $(5$ mg) & \\
\hline Nr. Cycles $(\geq 6)^{b}$ & $0.14[0.05-0.41]$ & $<.001$ \\
\hline Multivariate analysis & $0.90[0.28-2.92]$ & .863 \\
\hline Erythroid dysplasia & & \\
\hline Megakaryocytic dysplasia & $2.19[0.66-7.24]$ & .198 \\
\hline Initial dosage $(5$ mg) & & $<.001$ \\
\hline
\end{tabular}

ALIP, Abnormal Localisation of Immature Precursors; $\mathrm{Cl}$, confidence interval; $\mathrm{Hb}$, hemoglobin; $\mathrm{MCV}$, mean corpuscolar volume; OR, Odds ratio. ${ }^{a}$ Reference $10 \mathrm{mg}$ (either continuous or for $21 \mathrm{~d}$ ).

${ }^{\mathrm{b}}$ Reference $<6$ cycles.

with one more change and $8,1 \%$ with complex $5 q$-, List et $\mathrm{al}^{14}$ found AML evolution in $28.6 \%$ of cases 5 years after treatment initiation. Median time of progression was not reached for patients with isolated $5 \mathrm{q}$-, while it was 4.1 years for patients with additional cytogenetic abnormalities. A retrospective study reported no significant difference in the AML evolution rate in 125 untreated and 295 treated cases with isolated del $(5 q) .{ }^{15} \mathrm{AML}$ evolution was observed in $12.6 \%$ of 381 internationally recruited untreated cases with haematological and cytogenetic features similar to those in our study, namely low-int1 risk MDS, a low proportion of complex karyotypes (4.2\%) a low proportion of $5 q$ - plus another aberration (14.2\%), a median observation time of 49.8 months. $^{16}$

In this study, cases developing $\mathrm{AML}$ and those progressing to higher risk MDS were both negative for any cytogenetic response to LEN treatment. Clonal progression and development of a complex karyotype in 7/13 cases corresponded to AML development, whereas complex cytogenetics and new cytogenetic aberrations never appeared when MDS evolved towards a higher risk category. Although this observation needs to be confirmed in larger series of cases, our cytogenetic findings suggest that different biological features underlie disease progression to either worsening MDS or AML.

Unfortunately, biological samples were not available to evaluate molecular prognostic factors predictive of lower cytogenetic response or disease evolution, such as p53 expression or mutations, ${ }^{17,18}$ TET2, ASXL1, RUNX1 and CSNK1A1 mutations, ${ }^{19,20}$ or the expression of cereblon, whose reduction correlates with LEN resistance. $^{21}$

In conclusion, this "real life" Registry showed that LEN treatment successfully achieved a high erythroid response rate and reduced transfusion dependence in Low-Int1 risk MDS with del(5q), both as sole anomaly or associated with one change. The leukemic evolution rate was similar to that observed in other multi-centre studies. Cytogenetic results emphasised biological differences in cases with evolution to AML or to higher risk MDS. International efforts should be made to investigate predictive biological markers in large-scale clinical studies.

\section{AUTHOR CONTRIBUTIONS}

FA, AR, VDB, AC, CM collected, assembled, analysed and interpreted the data. CM, SC and ST conceived and designed the study. FA, AR, VDB and CM wrote the Article. All Authors provided final approval.

\section{ORCID}

Valeria Di Battista (iD http://orcid.org/0000-0003-3641-3643

\section{REFERENCES}

1. List A, Kurtin S, Roe DJ, et al. Efficacy of lenalidomide in myelodysplastic syndromes. N Engl J Med. 2005;352:549-557.

2. List A, Dewald G, Bennett J, et al. Lenalidomide in the myelodysplastic syndrome with chromosome $5 q$ deletion. N Engl J Med. 2006;355:1456-1465.

3. Fenaux P, Giagounidis A, Selleslag D, et al. A randomized phase 3 study of lenalidomide versus placebo in RBC transfusion dependent patients with low/Intermediate-1-risk myelodysplastic syndromes with del 5q. Blood. 2011;118:3765-3776. prepublished online.

4. Greenberg P, Cox C, LeBeau MM, et al. International scoring system for evaluating prognosis in myelodysplastic syndromes. Blood. 1997;89:2079-2088.

5. Cheson BD, Greenberg PL, Bennett JM, et al. Clinical application and proposal for modification of the International Working Group (IWG) response criteria in myelodysplasia. Blood. 2006;108:419-425.

6. Giagounidis AA. Lenalidomide for del( $5 q)$ and non-del( $5 q)$ myelodysplastic syndromes. Semin Hematol. 2012;49:312-322.

7. Le Bras F, Sebert M, Kelaidi C, et al. Treatment by Lenalidomide in lower risk myelodysplastic syndrome with $5 q$ deletion the GFM experience. Leuk Res. 2011;35:1444-1448.

8. Giagounidis A, Mufti GJ, Fenaux P, Germing U, List A, MacBeth KJ. Lenalidomide as a disease-modifying agent in patients with del( $5 q)$ myelodysplastic syndromes: linking mechanism of action to clinical outcomes. Ann Hematol. 2014;93:1-11.

9. Zeidan AM, Gore SD, McNally DL, et al. Lenalidomide performance in the real world: patterns of utilization of effectiveness in a medicare population with Myelodysplastic Syndromes. Cancer. 2013;119:3870-3878

10. Schuler E, Giagounidis A, Haase D, et al. Results of a multicenter prospective phase II trial investigating the safety and efficacy of lenalidomide in patients with myelodysplastic syndromes with isolated del(5q) (LE-MON 5). Leukemia. 2015;30:1580-1582.

11. Mallo M, Del Rey M, Ibáñez M, et al. Response to lenalidomide in myelodysplastic syndromes with del( $5 q)$ : influence of cytogenetics and mutations. Br J Haematol. 2013;162:74-86. 
12. Bennett JM. Changes in the updated 2016: WHO classification of the myelodysplastic syndromes and related myeloid neoplasm. Clin Lymphoma Myeloma Leuk. 2016;16:607-609.

13. Raza A, Reeves JA, Feldman EJ, et al. Phase 2 study of lenalidomide in transfusion-dependent, low-risk, and intermediate-1 risk myelodysplastic syndromes with karyotypes other than deletion $5 \mathrm{q}$. Blood. 2008;111:86-93.

14. List AF, Bennett JM, Sekeres MA, et al. Extended survival and reduced risk of $A M L$ progression in erythroid-responsive lenalidomide-treated patients with lower-risk del(5q) MDS. Leukemia. 2014;28:1033-1040.

15. Kuendgen A, Lauseker M, List AF, et al. Lenalidomide does not increase $A M L$ progression risk in RBC transfusion-dependent patients with Low- or Intermediate-1-risk MDS with del(5q): a comparative analysis. Leukemia. 2013;27:1072-1079.

16. Germing U, Lauseker M, Hildebrandt B, et al. Survival, prognostic factors and rates of leukemic transformation in 381 untreated patients with MDS and del(5q): a multicenter study. Leukemia. 2012;26:1286-1292.

17. Jädersten M, Saft L, Smith A, et al. TP53 mutations in low-risk myelodysplastic syndromes with del(5q) predict disease progression. J Clin Oncol. 2011;20:1971-1979.

18. Haferlach C, Dicker F, Herholz H, Schnittger S, Kern W, Haferlach T. Mutations of the TP53 gene in acute myeloid leukemia are strongly associated with a complex aberrant karyotype. Leukemia. 2008;22:1539-1541.

19. Scharenberg C, Giai V, Pellagatti A, et al. Progression in patients with low- and intermediate1-risk del(5q) myelodysplastic syndromes is predicted by a limited subset of mutations. Haematologica. 2017;102:498-508.

20. List A, Ebert BL, Fenaux P. A decade of progress in myelodysplastic syndrome with chromosome $5 q$ deletion. Leukemia. 2018; http:// doi.org/10.1038/s41375-018-0029-9. [Epub ahead of print]

21. Jonasova A, Bokorova R, Polak J, et al. High level of full-length cereblon mRNA in lower risk myelodysplastic syndrome with isolated $5 q$ deletion is implicated in the efficacy of lenalidomide. Eur J Haematol. 2015;95:27-34.

\section{SUPPORTING INFORMATION}

Additional Supporting Information may be found online in the supporting information tab for this article.

How to cite this article: Arcioni F, Roncadori A, Di Battista V, et al. Lenalidomide treatment of myelodysplastic syndromes with chromosome $5 q$ deletion: Results from the National Registry of the Italian Drug Agency. Eur J Haematol.

2018;101:78-85. https://doi.org/10.1111/ejh.13067

\section{APPENDIX \\ MORE Study Centres}

Azienda Ospedaliera Sant'Anna e San Sebastiano di Caserta, sezione di Oncoematologia (Antonio Abbadessa); Azienda Ospedaliera Universitaria Careggi di Firenze, sezione Ematologia (Renato Alterini, Valeria Santini); Policlinico Tor Vergata Roma, Sezione Ematologia, (Maria Cantonetti, Francesco Buccisano); Azienda Ospedaliero Universitaria San Martino di Genova, (Andrea Bacigalupo, Mario
Sessarego); Azienda Sanitaria Locale di Biella, Dipartimento di Medicina Interna e Urgenza (Anna Tonso); Ospedale Universitario Molinette San Giovanni Battista di Torino, Sezione Ematologia (Dario Ferrero, Stefano D'Ardia); Ospedale Mauriziano Umberto I, Torino (Corrado Tarella); Ospedale "Casa Sollievo della Sofferenza" IRCCS di S. Giovanni Rotondo, Sezione Ematologia (Nicola Cascavilla); Azienda ULSS 12 Veneziana, sezione Ematologia (Renato Bassan, Rosaria Sancetta); Fondazione IRCCS Ca' Granda Ospedale Maggiore Policlinico Milano, Sezione di Ematologia (Agostino Cortelezzi, Gianluigi Reda); Ospedale "A.Tortona" di Pagani, Medicina Interna e Oncoematologia (Alfonso Maria D'Arco); Ospedale Sant'Eugenio, Unità di Ematologia (Paolo De Fabritiis); Ospedale Vito Fazzi di Lecce, sezione Ematologia (Nicola Di Renzo); Università Degli Studi di Perugia, Sezione Ematologia (Brunangelo Falini); Ematologia Università "Sapienza" Roma (Giuliana Alimena); Arcispedale Santa Maria Nuova di Reggio Emilia, sezione Ematologia (Paolo Avanzini, Fiorella llariucci); Ospedale Nicola Gianettasio di Rossano Calabro, Cosenza, sezione Oncologia (Francesco luliano); ASL Cagliari Presidio Ospedaliero Roberto Binaghi, Sezione Ematologia (Giorgio La Nasa, Giovanni Caocci); Ospedale Policlinico Santa Maria alle Scotte di Siena, sezione Ematologia (Marzia Defina); Ospedale San Francesco di Nuoro, sezione Ematologia (Giancarlo Latte, Angelo Palmas); Azienda Ospedaliera Nazionale "SS. Antonio e Biagio e C. Arrigo" di Alessandria Struttura Complessa di Ematologia (Alessandro Levis); Policlinico Agostino Gemelli Roma, Ematologia (Giuseppe Leone, Maria Teresa Voso); Azienda Ospedaliero-Universitaria Ospedali Riuniti di Ancona, Sezione Ematologia (Pietro Leoni, Antonella Poloni); Azienda Ospedaliero Universitaria di Sassari, sezione Ematologia (Claudio Fozza); Azienda Ospedaliero Universitaria di Parma, Sezione Ematologia con Trapianto di Midollo Osseo (Monica Crugnola); ASL Viterbo, Stabilimento di Ronciglione, Day Hospital di Ematologia (Marco Montanaro); Azienda Socio Sanitaria Territoriale di Cremona, Ematologia (Pierangelo Spedini, Francesco Lanza); Azienda Ospedaliera di Potenza, Ematologia (Michele Pizzuti); Divisione di Ematologia - Dipartimento di Medicina Clinica e Chirurgica Università Federico II di Napoli (Fabrizio Pane); Azienda ULSS 18 Rovigo, Sezione Ematologia (Rossella Paolini); Clinica Ematologica CTA,Università degli Studi Milano Bicocca - Azienda Ospedaliera San Gerardo Monza (Lorenza Borin); Azienda Ospedaliera Papa Giovanni XXIII, Sezione di Ematologia, Bergamo (Alessandro Rambaldi); Presidio Ospedaliera di Brescia sezione Ematologia (Giuseppe Rossi, Anna Maria Pelizzari); Spedali Civili di Brescia sezione Trapianto di Midollo Osseo (Domenico Russo); Azienda ULSS 6 Vicenza, Ematologia (Anna D’Emilio, Marco Ruggeri); Azienda Ospedaliera di Padova, sezione di Ematologia (Giampietro Semenzato); Azienda Ospedaliera Policlinico Consorziale BARI, Ematologia Universitaria (Giorgina Specchia); USL Asolo, Sezione di Immunoematologia e Trasfusionale (Giuseppe Tagariello, Roberto Sartori); Ospedale Cardinal Massaia di Asti sezione Oncologia (Franco Testore, Giorgio Ciravegna); Policlinico di Modena, sezione Ematologia (Roberto Marasca); ULSS 1 Belluno, Sezione di Medicina Interna (Lorella Cimarosto, Orietta Fontanive); Ospedali Riuniti Pesaro, Ematologia e Centro Trapianti (Giuseppe Visani). 